\title{
Azole Resistance in Candida Species Isolated from Diabetic Women with Vulvovaginal Candidiasis in a Tertiary Care Centre
}

\author{
L. Gracia Paul*, I.M. Rejitha and C. Revathy \\ Department of Microbiology, Tirunelveli Medical College, Tirunelveli - 627 011, \\ Tamil Nadu, India \\ *Corresponding author
}

\section{Keywords \\ Vulvovaginal candidiasis, Diabetes mellitus, Non-albicans Candida, Fluconazole resistance \\ Article Info \\ Accepted: \\ 07 January 2019 \\ Available Online: \\ 10 February 2019}

\section{A B S T R A C T}

Candidiasis has emerged as an alarming opportunistic infection with an increase in a number of patients among the diabetics. It is estimated that as many as $75 \%$ of women experience at least one episode of Vulvovaginal Candidiasis during their lifetime. Over the last decade, non-albicans Candida associated with vulvovaginal infections have increased and in recent times have developed resistance to anti- fungal agents, in particular to the azole compounds. This prospective study was carried out to determine the relative contribution of Candida abicans and non-albicans species in diabetic patients with vulvovaginal candidiasis and their antifungal susceptibility profile. 56 samples collected from diabetic females with symptomatic vulvovaginitis were gram stained and cultured on to Sabourads Dextrose agar and then speciated. Antifungal susceptibility testing of these isolates was done by Kirby-Bauer disk diffusion method. $41.1 \%$ of diabetic females had Vulvovaginal candidiasis among which $39.1 \%$ were Candida albicans and $61.9 \%$ were non-albicans Candida. Among the non-albicans Candida isolates, Candida tropicalis and Candida glabrata were the major isolates. The antifungal susceptibility testing of isolates showed that $39.1 \%$ were sensitive, $34.8 \%$ were susceptible dose dependent (SSD) and $17.4 \%$ were resistant to Fluconazole while most of the isolates were sensitive to Voriconazole and all the isolates were sensitive to Itraconazole. Among $17.4 \%$ of Fluconazole resistant isolates, $75 \%$ were Candida tropicalis and $25 \%$ were Candida albicans. This study shows that the isolation of non-albicans Candida have clinical implications due to their reduced susceptibility to various antifungals, thus highlighting the importance of isolation, species identification and antifungal susceptibility testing prior to initiation of therapy for proper selection of antifungal agent. The judicious use of antifungal agents is very important for the prevention of emergence and spread of drug resistant Candida species.

\section{Introduction}

Candida species are commonly seen fungi that exist as an element of normal flora in the skin, gastrointestinal tract and reproductive tract of humans. Fungal infections are generally opportunistic so the pathogenesis and prognosis of candidial infections are affected by the host immune status and also differ greatly according to disease presentations (1). Vulvovaginal candidiasis (VVC) is the most common manifestation of 
genital candidiasis. It is defined as signs and symptoms of inflammation of the vulva and vagina in the presence of Candida species. Clinical manifestations of VVC are pruritus, hyperemia, vaginal discomfort, leucorrhea, burning, soreness, dyspareunia and vaginal or vulvar erythema. VVC is diagnosed in upto $40 \%$ of females with vaginal complaints (2).

VVC is the second most common after bacterial vaginosis among the many causes of vaginitis and it is estimated that approximately $75 \%$ of all women suffer at least once in their lifetime from vulvovaginal candidiasis (VVC), with 40-50\% experiencing at least one additional episode of infection. A small percentage of women (5$8 \%$ ) suffer from at least four recurrent VVC per year (3). The prevalence of Candida infections accounts for about $47.5 \%$ among diabetic women.

Candida albicans is the cause of VVC in majority of cases, but recent studies document the increasing episodes of vulvovaginitis due to non albicans Candida (NAC) species.VVC due to NAC spp. is clinically indistinguishable from that caused by $C$. albicans and is more resistant to antifungal treatment. The use of single dose oral and topical formulation together with low dosage azole maintenance regimen and the availability of over-the-counter antifungals are risk factors suggested for the increase of VVC due to NAC species.

Diabetes mellitus is a chronic, insidious disease that can affect any organ or system of the body and one of the major complications associated with it is infection. Although the prevalence of infection among diabetic and non-diabetic subjects is similar, the intensity of infection is being more severe and the response to therapy is slow in diabetic patients. Diabetes is a proven predisposing factor for the imbalance in vaginal micro flora resulting in vulvovaginal candidiasis Symptomatic vulvovaginal candidiasis has been proven to be more prevalent in diabetic women than in the general population.

A number of antifungal agents especially azoles are used to treat candidiasis. Candida albicans and related species in the recent times have developed resistance to anti- fungal agents, in particular to the azole compounds. Currently, Fluconazole is recommended in various guidelines as the first drug of choice because it is less toxic and can be taken as a single oral dose (4). Emergence of Fluconazole-resistant Candida species has been progressively reported in the last few years. Hence, accurate species identification is important for the treatment of Candida infections, as the non-albicans Candida continue to be increasingly documented.

This study was done to isolate and speciate Candida isolates from diabetic patients with symptomatic Vulvovaginal candidiasis and to assess the anti fungal susceptibility profile of the isolated species of Candida.

\section{Materials and Methods}

This prospective study was conducted at Tirunelveli Medical College, Tamilnadu from June 2017 to July 2018 to isolate, speciate and determine the antifungal susceptibility of Candida isolates obtained from diabetic women with vulvovaginal candidiasis. This study was started after getting ethical committee clearance from the institution.

Inclusion Criteria: Diabetic patients with symptomatic vulvovaginitis.

Exclusion Criteria: Patients who were not suffering from diabetes, Pregnant women, patients who were on cancer chemotherapy and antibiotics and patients suffering from 


\section{HIV/AIDS}

Details like name, age, sex, Fasting blood glucose levels, treatment status for diabetes and other parameters significant to the present study were collected. Vulvovaginal swabs (2 swabs for each patient) collected using cotton tipped swabs were immediately transported to the Microbiology Laboratory of the hospital for processing.

One swab was used for the direct microscopic examination by Gram stain and screened for presence of budding yeast cells and pseudohyphae. Second swab was inoculated immediately into Sabourads dextrose agar plates and incubated at $37^{\circ} \mathrm{C}$ for 24-72 hours. Isolates were identified by colony morphology and further confirmed by Gram stain.

Isolates were speciated by Germ tube test, Dalmau plate method, growth at $42^{\circ} \mathrm{C}$, Candida CHROM agar and sugar assimilation tests. Antifungal susceptibility testing of the isolates were done by disk diffusion method according to The National Committee for Clinical Laboratory Standards (NCCLS) Method for Antifungal Disk Diffusion Susceptibility Testing of Yeasts; Approved Guideline. NCCLS document M44-A using Fluconazole $(25 \mu \mathrm{g})$, Voriconazole $(1 \mu \mathrm{g})$ and Itraconazole $(10 \mu \mathrm{g})$. The antifungal susceptibility of the isolates was interpreted as sensitive (S), dose dependent-susceptible (DDS) and resistant (R) according to the zone diameters.

\section{Statistical analysis}

Data collected were entered in Microsoft Excel and analysed using statistical analysis software Statistical Package for Social Services (SPSS) v.16. Appropriate statistics were calculated. $\mathrm{p}$ values $<0.05$ were statistically significant.

Results and Discussion
The mean age of the 56 diabetic women from whom samples were collected was found to be 47 years with SD 8.9 years and the most common symptom among the study group was found to be vaginal discharge. None of the diabetic population among the study population had fasting blood glucose level under control (i.e. $<126 \mathrm{mg} / \mathrm{dl}$ ). The mean fasting blood glucose level of the diabetic patients was found to be $152 \mathrm{mg} / \mathrm{dl}$ (SD 11 $\mathrm{mg} / \mathrm{dl})$. Fasting blood glucose level showed a statistically significant relationship with the age of the diabetic patients. This study shows that the FBS levels steadily increases with increase in age of the diabetic patients ( $p$ value $<0.001$, One way ANOVA) and only $37 \%$ of the study group was on therapy for diabetes.

On gram staining, only $27 \%$ (15 smears) were found to be positive for Candida of which $20 \%$ showed budding yeast cells with pseudohyphae and the rest showed budding yeast cells only. From the 56 processed samples, 23(41\%) Candida isolates were obtained. The culture results for Candida did not show any significant association with age and fasting blood glucose levels of the diabetic patients. However, there is significant relationship between the culture results and the treatment status of the patients. Culture results showed that Candida was isolated from nearly $41 \%$ of the specimens collected from the study group, whereas gram staining found only $27 \%$ of the specimens to be positive for Candida.

Speciation of Candida was done by using CHROM agar, Dalmau plate technique, Germ tube test, Growth at $42^{\circ} \mathrm{C}$ and sugar assimilation tests. $39.1 \%$ of the culture positive isolates were found to be Candida albicans and the remaining $61 \%$ were found to be non-albicans Candida. Of the total isolates, $26.1 \%$ were C. tropicalis, $17.4 \%$ were $C$. parapsilosis, $8.7 \%$ were $C$. glabarata 
and $8.7 \%$ were $C$. krusei as shown in Table 1 .

All the 23 Candida isolates were subjected to antifungal susceptibility testing by disc diffusion method using Fluconazole $(25 \mu \mathrm{g})$, Voriconazole $(1 \mu \mathrm{g})$ and Itraconazole $(10 \mu \mathrm{g})$. Highest level of sensitivity (100\%) was seen for Itraconazole followed by Voriconazole. Highest level of resistance (17.4\%) was seen against Fluconazole. 22 isolates were sensitive to Voriconazole and one isolate was susceptible dose dependent to Voriconazole. The Fluconazole susceptibility pattern of the isolates was shown in Table 2. The 4 Fluconazole resistant isolates were isolated from patients who were not on any form of treatment for diabetes. Among the 4 isolates, one of them was Candida albicans $(25 \%)$ and the rest were Candida tropicalis (75\%).

Table.1 Distribution of Candida species among female diabetics with Vulvovaginal candidiasis

\begin{tabular}{|c|c|c|}
\hline \multirow{2}{*}{ Species } & \multicolumn{2}{|c|}{ Vulvovaginal candidiasis } \\
\cline { 2 - 3 } & No. & $\%$ \\
\hline Candida albicans & 9 & 39.1 \\
\hline Candida tropicalis & 6 & 26.1 \\
\hline Candida glabrata & 4 & 17.4 \\
\hline Candida parapsilosis & 2 & 8.7 \\
\hline Candida krusei & 2 & 8.7 \\
\hline
\end{tabular}

Table.2 Fluconazole susceptibility pattern of isolates from Vulvovaginal Candidiasis

\begin{tabular}{|c|c|c|c|}
\hline Candida species & Sensitive & $\begin{array}{c}\text { Susceptible Dose } \\
\text { Dependent }\end{array}$ & Resistant \\
\hline Candida albicans & $6(66.7 \%)$ & $2(22.2 \%)$ & $1(11.1 \%)$ \\
\hline Candida tropicalis & $2(33.3 \%)$ & $1(16.7 \%)$ & $3(50 \%)$ \\
\hline Candida glabrata & - & $4(100 \%)$ & - \\
\hline Candida parapsilosis & $1(50 \%)$ & $1(50 \%)$ & - \\
\hline
\end{tabular}

Candidiasis has emerged as an alarming opportunistic infection with an increase in a number of patients among the diabetics. Over the last decade, non-albicans Candida associated with human infections have increased and in recent times have developed resistance to anti- fungal agents, in particular to the azole compounds. The present study was carried out to determine the relative contribution of $C$. abicans and non-albicans species in diabetic patients with vulvovaginal candidiasis and their antifungal susceptibility pattern.
In our study, 56 vaginal specimens were collected from diabetic females with symptomatic vulvovaginitis. The patients in our study had a mean age of 47 years (with SD 8.9 years) and $42.9 \%$ fell between 41-50 years of age. In our study, the chief complaint among the symptomatic female diabetic patients was vaginal discharge which accounts for about $46.4 \%$ followed by pruritis and burning sensation (35.7\%). These results were in accordance with the study by Rathod et al., which showed a higher rate of complaints of vaginal discharge $(31 \%)$ followed by pruritis (29\%) (5). Both Vaginal 
discharge and pruritis with burning sensation were present in $17.9 \%$ of the diabetic females. Out of the 56 diabetic females, only three $(5.4 \%)$ complained of recurrence of symptoms within one year.

The positive predictive values of these symptoms for predicting vulvovaginal candidiasis were low. A minority of women with these symptoms were subsequently diagnosed with vulvovaginal candidiasis (53.85\%, 25\% and 50\% respectively). The low positive predictive value of symptoms indicate that the patients should not be treated based on symptoms alone which may inadvertently lead to treatment of patients without arriving at a correct diagnosis.

The mean fasting blood glucose level of the diabetic patients was found to be $152 \mathrm{mg} / \mathrm{dl}$ (SD $11 \mathrm{mg} / \mathrm{dl}$ ). Fasting blood glucose level showed a statistically significant relationship with the age of the diabetic patients. This study also shows that the FBS levels steadily increases with increase in age of the diabetic patients and there is a significant positive correlation between the age of the patient and FBS levels.

$73 \%$ of the vaginal smears were negative on gram staining and only $26.8 \%$ were found to be positive which is higher than that of the study by Vijaya et al., which showed a positivity of only $7.3 \%$ (6). Culture positivity for Candida was found in $41.1 \%$ of diabetic females in our study. Studies by Goswami et $a l$., and Shrivastav et al., showed a culture positivity of $46 \%$ and $47.5 \%$ respectively $(7,8)$. Peer et al., reported a prevalence rate of $35.5 \%$ and Faraji et al.,. reported a prevalence of vulvovaginal candidiasis as $20 \%$ among diabetic women in their studies $(9,10)$.

The culture positivity for Candida did not show any significant association with age and fasting blood glucose levels of the diabetic patients but there is a significant relationship between the occurrence of candidiasis and the treatment status of patients for diabetes. This study shows that $26.32 \%$ of diabetic females who were on treatment and $48.65 \%$ who were not on any form of treatment for diabetes suffered from vulvovaginal candidiasis respectively.

The species obtained from vulvovaginal candidiasis were Candida albicans (39.1\%), Candida tropicalis (26.1\%), Candida glabrata (17.4\%), Candida parapsilosis $(8.7 \%)$ and Candida krusei (8.7\%). The distribution of Candida species isolated from diabetic patients with vulvovaginal candidiasis showed a majority of Candida non albicans which was $61.9 \% \quad(\mathrm{n}=14)$ whereas Candida albicans isolates constituted only $39.1 \%(\mathrm{n}=9)$. Among the non albicans Candida $(\mathrm{n}=14)$, Candida tropicalis was the commonest isolate $42.85 \%(n=6)$ in our study followed by Candida glabrata $28.57 \%(\mathrm{n}=4)$, Candida parapsilosis $14.29 \% \quad(\mathrm{n}=2)$ and Candida krusei $14.29 \%(\mathrm{n}=2)$ whereas studies by Shrivastav et al., Faraji et al., and Ray D et al., showed that the predominant Candida species isolated in diabetics with vulvovaginal candidiasis was Candida glabrata $(8,10,11)$.

The antifungal susceptibility testing done by Kirby-Bauer disk diffusion method on isolates from Vulvovaginal candidiasis showed that $39.1 \% \quad(n=9)$ were sensitive, $34.8 \% \quad(n=8)$ were susceptible dose dependent (SSD) and $17.4 \%(n=4)$ were resistant to Fluconazole in our study. Candida krusei which constituted $8.7 \%(n=2)$ of the isolates were considered resistant as they are intrinsically resistant to Fluconazole. $22(95.7 \%)$ isolates were sensitive to Voriconazole and only one isolate (4.3\%) which was Candida krusei showed intermediate resistance. All the isolates were sensitive to Itraconazole. Studies by 
Kalaiarasan et al., and Khadka $\mathrm{S}$ et al., showed a higher susceptibility to Fluconazole compared to our study $(12,13)$ whereas a study by Khan $\mathrm{M}$ et al., showed a lower susceptibility of isolates to Fluconazole(14). The increased resistance and dose dependent susceptibile isolates in our study compared to the above studies may be attributed to the presence of large number of Candida glabrata and Candida tropicalis in a small number of isolates.

Among the Candida albicans isolates, $66.7 \%$ were sensitive, $22.2 \%$ were susceptible dose dependent and only $11.1 \%$ were resistant to Fluconazole whereas among NAC species only $21.43 \%$ were sensitive, $42.86 \%$ were susceptible dose dependent, $21.43 \%$ were resistant and the rest were intrinsically resistant to Fluconazole. 33.3\%, 16.7\% and $50 \%$ of Candida tropicalis isolates were sensitive, SDD and resistant to Fluconazole respectively. These finding are contrary to the study by Khan $\mathrm{M}$ et al., which showed that Fluconazole susceptibility was less in Candida albicans isolates compared to non albicans Candida isolates (14). All the isolates of Candida glabrata were reported as SDD as they cannot be reported sensitive to Fluconazole as per CLSI guidelines which is similar to study by Mohanty et al., (15). Among the Candida parapsilosis isolates half were sensitive and the rest were SDD to Fluconazole. This study shows that majority of the SDD and resistant isolates were nonalbicans Candida compared to Candida albicans. Among $17.4 \%$ of Fluconazole resistant isolates, $75 \%$ were Candida tropicalis and $25 \%$ were Candida albicans which is similar to the results obtained in a study by Deorukhkar et al., (2).

In conclusion, the present study demonstrated the importance of species identification and susceptibility testing for antifungals in Candida isolated from diabetic patients as uncontrolled diabetes mellitus causes metabolic alterations such as increased levels of glycogen, which can significantly increase colonization and infection by Candida. Isolation of non-albicans Candida have clinical implication due to their reduced susceptibility to various antifungals, thus highlighting the importance of isolation, species identification and antifungal susceptibility of Candida prior to initiation of therapy for proper selection of antifungal agent. The judicious use of antifungal agents is very important for the prevention of emergence and spread of drug resistant Candida species. Antifungal susceptibility testing should be used to calculate clinical response and accordingly local antibiograms can aid in empirical assortment of antifungals, guiding options for long-term therapy and alternative regimens in testing of isolates from recurrent infections.

Acknowledgement: The authors gratefully acknowledge The Dean, Tirunelveli Medical College Hospital, Tirunelveli, Tamil Nadu, The Staff of Microbiology Department, Obstetrics and Gynaecology Department of Tirunelveli Medical College Hospital.

\section{References}

1. Iven PC. Mycotic diseases. In: McPherson RA, Pincus MR (Eds.), Henry's Clinical Diagnosis and Management by Laboratory Methods. 22nd ed. Philadelphia: Saunders Elsevier; 2011. p. 1155-87.

2. Deorukhkar SC, Santosh Saini S, Mathew S. 2014. Nonalbicans Candida infection: an emerging threat. Interdisciplinary Perspect. Infect. Dis. 2014: 615958.

3. Sobel JD. Candidal vulvovaginitis. Clin Obstet Gynecol 1993; 36: 153-212

4. Centers for Disease Control and Prevention (CDC). Sexually Transmitted Diseases Treatment Guidelines 2002; 
http://www.cdc.gov/std/treatment/default. htm.

5. Rathod SD, Klausner JD, Krupp K, Reingold AL, Madhivanan P, Epidemiologic Features of Vulvovaginal Candidiasis among Reproductive-Age Women in India Infect Dis Obstet Gynecol 2012 2012:859-71.

6. Vijaya D, Dhanalakshmi TA, Kulkarni S. Changing Trends of Vulvovaginal Candidiasis. Journal of Laboratory Physicians. 2014; 6(1): 28-30. doi:10.4103/0974-2727.129087.

7. Goswami R, Dadhwal V, Tejaswi S, Datta K, Paul A, Haricharan RN, Banerjee U, Kochupillai N., 2000. Species specific prevalence of Vaginal candidiasis among patients with diabetes mellitus and its relation to their glycaemic status. Infect. J., 41(2):6-162

8. Shrivastav V K, Shukla D, Shrivastav A, Jana AM. Prevalence of vaginal candidiasis in diabetic women of Madhya Pradesh, India. Int J Curr Microbiol App Sci. 2015; 4(5): 834-46.

9. Peer AK, Hoosen AA, Seedat MA, vanden- Ende J, Omar MA., 1993. Vaginal yeast infections in diabetic women. Afr. $J$. Med., 83: 9-727.

10. Faraji R, Rahimi MA, Rezvanmadani F, Hashemi M. 2012. Prevalence of vaginal candidiasis infection in diabetic women. African. J. Microbiol. 6: 2773-2778
11. Ray D, Goswami R, Banerjee U, Dadhwal V, Goswami D, Mandal P, et al., 2007. Prevalence of Candida glabrata and its response to boric acid suppositories in comparison with oral Fluconazole in patients with diabetes and vulvovaginal candidiasis. Diabetes Care 30: 312-317.

12. Kalaiarasan K, Singh R, Chaturvedula L. Fungal profile of vulvovaginal candidiasis in a tertiary care hospital. $\mathrm{J}$ Clin Diagn Res. 2017 Mar; 11(3): DC06DC09.

13. Khadka S, Sherchand JB, Pokhrel BM, et al., Isolation, speciation and antifungal susceptibility testing of Candida isolates from various clinical specimens at a tertiary care hospital, Nepal. $B M C$ Research Notes. 2017; 10: 218.

14. Khan M, Ahmed J, Gul A, Ikram A, Lalani FK. Antifungal susceptibility testing of vulvovaginal Candida species among women attending antenatal clinic in tertiary care hospitals of Peshawar. Infection and Drug Resistance. 2018; 11: 447-456.

15. Mohanty S, Xess I, Hasan F, Kapil A, Mittal S, Tolosa JE, Prevalence and susceptibility to Fluconazole of candida species causing vulvovaginitis Indian $J$ Med Res. 2007; 126: 216-9.

\section{How to cite this article:}

Gracia Paul, L., I.M. Rejitha and Revathy, C. 2019. Azole Resistance in Candida Species Isolated from Diabetic Women with Vulvovaginal Candidiasis in a Tertiary Care Centre. Int.J.Curr.Microbiol.App.Sci. 8(02): 738-744. doi: https://doi.org/10.20546/ijcmas.2019.802.085 